\title{
Deep venous thrombosis and pulmonary embolism after COVID-19 mRNA vaccination
}

\author{
Ali Atoui ${ }^{1} \cdot$ Kawthar Jarrah ${ }^{1} \cdot$ Layal Al Mahmasani $^{1} \cdot$ Rayan Bou-Fakhredin $^{2} \cdot$ Ali T. Taher $^{1}$ (i)
}

Received: 29 November 2021 / Accepted: 14 December 2021 / Published online: 27 January 2022

(c) The Author(s), under exclusive licence to Springer-Verlag GmbH Germany, part of Springer Nature 2021

\section{Dear Editor,}

The COVID-19 pandemic has been deemed a global health emergency. Vaccination is considered the most important and effective strategy to prevent infection and eradicate the disease [1-3]. Since then, there is a significant interest in venous thromboembolism (VTE) and thrombocytopenia after COVID-19 vaccination. To date, there is only one report outlining a possible association between VTE and the COVID-19 mRNA vaccine days after the second dose in a patient with heterozygous Factor V Leiden (FVL) mutation [4]. Herein, we report a case of VTE and pulmonary embolism (PE) after administration of the COVID-19 mRNA vaccine as an acute phase reaction in a patient with thrombophilia.

A 24-year-old male patient was presented to our Hematology clinic for management of a recently diagnosed right lower extremity deep venous thrombosis (DVT) and PE. His medical history is unremarkable, with no previous thrombotic events reported. Two months earlier, he had received the second dose of Pfizer BioNTech mRNA COVID vaccine. Twenty-four hours post vaccination, the patient started to experience left sided pleuritic chest pain which was not relieved by paracetamol. The pain was associated with lowgrade fever and right lower extremity edema, erythema, and pain with inability to bear weight on right leg. Upon presentation to the emergency room, laboratory studies showed elevated D-dimer of $800 \mathrm{ng} / \mathrm{ml}$. Other blood tests (including complete blood count, international normalized ratio (INR), partial thromboplastin time (PTT), renal and hepatic panel)

Ali T. Taher

ataher@aub.edu.lb

1 Division of Hematology-Oncology, Department of Internal Medicine, American University of Beirut Medical Center, P.O. Box 11-0236, Beirut 11072020, Lebanon

2 Department of Clinical Sciences and Community Health, University of Milan, Milan, Italy were normal. A venous duplex ultrasound of lower extremities showed thrombophlebitis extending from the right external iliac vein to the common femoral vein reaching the right popliteal vein with a fresh thrombus at the common femoral vein. A computed tomography scan of the chest with intravenous contrast showed the presence of bilateral filling defects in the right superior and inferior lobar arteries and in the lingular and left inferior lobar arteries. An echocardiography showed normal right ventricle and ejection fraction of $64 \%$. The patient was started on enoxaparin $1 \mathrm{mg} / \mathrm{kg}$ every $12 \mathrm{~h}$ and admitted to the intensive care unit for observation. Thrombophilia screening showed FVL G169A heterozygous mutation and MTHFR A1298C homozygous mutation. His homocysteine level was also elevated $(254 \mathrm{umol} / \mathrm{L})$ with low folic acid $(1 \mathrm{ng} / \mathrm{ml})$ and vitamin B12 $(123 \mathrm{pg} / \mathrm{ml})$. There was no evidence of lupus anticoagulant. The patient was discharged home after few days on apixaban $5 \mathrm{mg}$ twice daily.

Thrombophilia is a blood coagulation disorder that is associated with a higher risk of VTE and is identified in almost half of patients presenting with thrombosis [5]. The prevalence of $\mathrm{FVL}$ is about $3-7 \%$ in the general population with a relative risk of 3 to 5 for first VTE compared to the normal population, and the presence of MTHFR mutations was found to be a combined risk factor for hypercoagulability disorders $[6,7]$.

The Pfizer BioNTech vaccine was the first approved vaccine for the prevention of COVID-19 infection, with an efficacy reaching $95 \%$ and a favorable safety profile and no VTE cases reported [3]. However, the safety of this vaccine has not been investigated in patients with inherited thrombophilia and who are at increased risk of VTE. The trigger of VTE in our patient could be attributed to a very intense immunological response secondary to the second dose of the vaccine, a pathophysiological mechanism commonly identified in several clinical conditions such as infections, sepsis, and inflammation[8]. However, the presence of the FVL mutation has been shown to enhance the thrombophilia tendency during an inflammatory episode [9]. The co-presence 


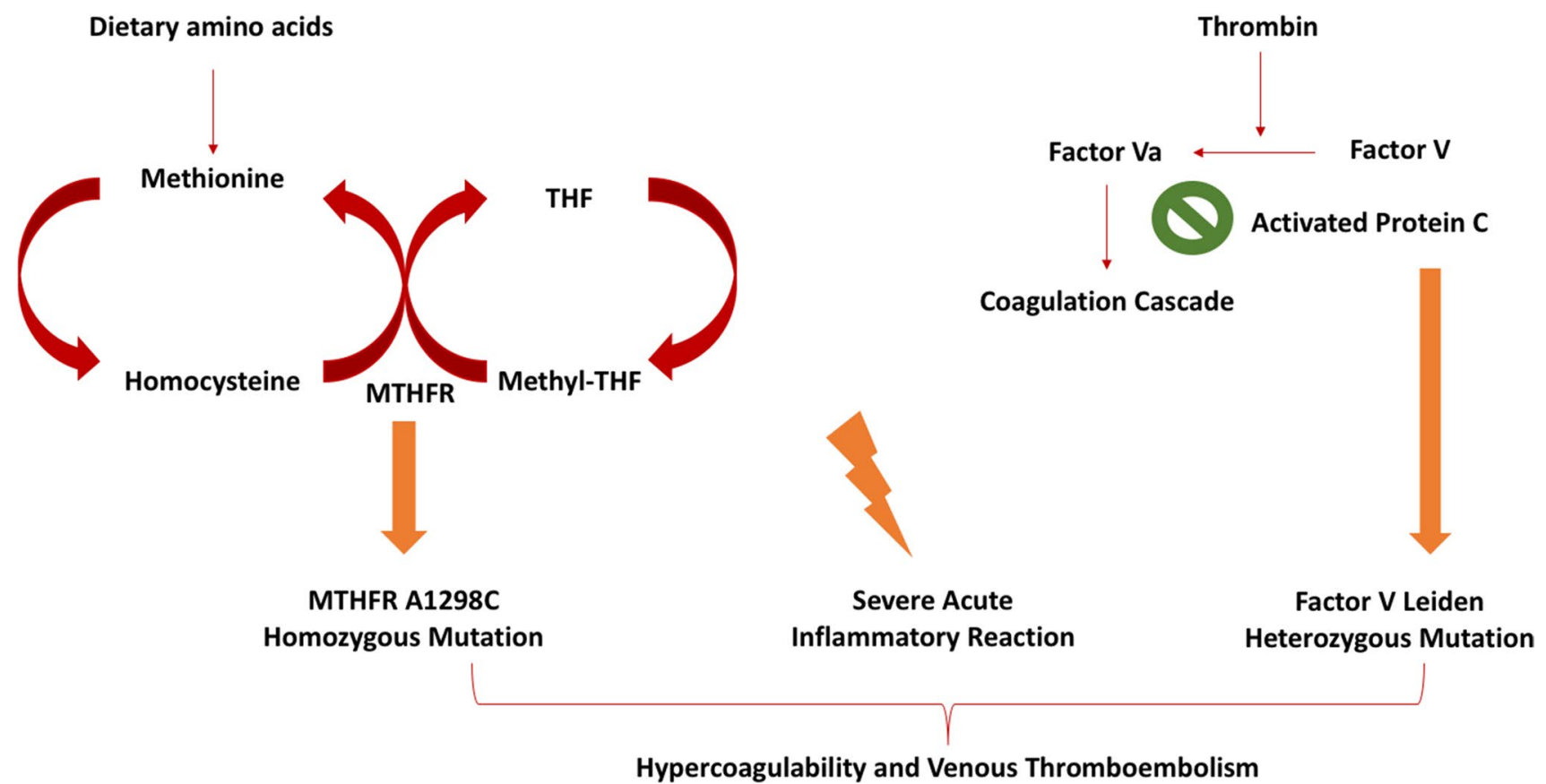

Fig. 1 Proposed pathophysiology of VTE after COVID-19 vaccination in the presence of hypercoagulable state. The combination of FVL heterozygous mutation leading to resistance to activated protein C, MTHFR A1298C homozygous mutation leading to increased

of the MTHFR mutation might have caused the increased risk of VTE in this patient. Multiple studies investigating the association of MTHFR mutations and VTE have shown conflicting results. In a pooled analysis investigating the association between different genetic alterations and VTE, the risk of VTE was increased in patients with homozygous C677T MTHFR mutation who are heterozygous for FVL [9]. Venous thromboembolism such as portal vein thrombosis has also been reported in a patient with heterozygous A1298A MTHFR mutation [10]. The association of all these factors including heterozygous FVL, homozygous MTHFR, and a robust inflammatory response after the vaccine could explain the occurrence of VTE in our patient (Fig. 1).

Interestingly, there have been serious concerns regarding an increased risk for varying types of thrombosis following vaccination for the COVID-19 virus including cerebral venous sinus thrombosis, arterial thrombosis, and splanchnic vein thrombosis [11]. One possible explanation could be related to the fact that antibodies against platelet factor 4 (PF4), as seen in heparin induced thrombocytopenia (HIT), form post vaccination and in turn contribute further to coagulopathy. In a case report describing a case of DVT and PE 7 days following the first dose of Pfizer BioNTech mRNA vaccine, laboratory tests were significant for positive HIT ELISA test with an elevated optical density assay of 0.617 [11]. Another study assessing the frequency of PF4 antibodies after COVID homocysteine levels, and a severe acute inflammatory reaction after mRNA COVID-19 vaccine could be a trigger for venous thromboembolism

vaccination showed 5.6\% antibodies with Pfizer BioNTech vaccine compared to $8 \%$ with Astrazeneca ChAdOx1vaccine. However, the clinical relevance of these antibodies was very minor [12].

In conclusion, we report a case of DVT and PE in a patient with a previously unknown FVL heterozygous mutation and MTHFR homozygous mutation, hours after the second dose of the Pfizer BioNTech mRNA COVID-19 vaccine. The COVID-19 mRNA vaccine has shown excellent efficacy with a favorable safety profile. However, we believe that in patients who are at an increased risk of thrombophilia especially those who carry multiple genetic abnormalities, the risk of VTE might be further increased due to the combination of these factors with an immune inflammatory response to the vaccine. It is advisable that patients with a previous history of unprovoked DVT or $\mathrm{PE}$, who were not tested for genetic risk, to be offered testing prior to COVID-19 vaccination. Currently, there is no sufficient data to support the exemption of these patients from vaccination or to use anticoagulation prophylaxis. However, to date, mRNA vaccines appear to be safe, and anticoagulation should be discussed on a case-by-case basis. Clinicians shall be aware of this increased risk, and patients shall be well educated on benefits and possible risks behind the vaccine. 
Author contribution All authors contributed to manuscript drafting or critical review and final approval for submission.

\section{Declarations}

Conflict of interest The authors declare no competing interests.

Informed consent The patient gave informed consent.

\section{References}

1. Voysey M, Clemens SAC, Madhi SA, Weckx LY, Folegatti PM, Aley PK et al (2021) Safety and efficacy of the ChAdOx1 nCoV19 vaccine (AZD1222) against SARS-CoV-2: an interim analysis of four randomised controlled trials in Brazil, South Africa, and the UK. Lancet (London, England) 397(10269):99-111

2. Baden LR, El Sahly HM, Essink B, Kotloff K, Frey S, Novak R et al (2021) Efficacy and safety of the mRNA-1273 SARS-CoV-2 vaccine. N Engl J Med 384(5):403-416

3. Polack FP, Thomas SJ, Kitchin N, Absalon J, Gurtman A, Lockhart S et al (2020) Safety and efficacy of the BNT162b2 mRNA Covid-19 vaccine. N Engl J Med 383(27):2603-2615

4. Carli G, Nichele I, Ruggeri M, Barra S, Tosetto A (2021) Deep vein thrombosis (DVT) occurring shortly after the second dose of mRNA SARS-CoV-2 vaccine. Intern Emerg Med 16(3):803-804

5. Cohn DM, Roshani S, Middeldorp S (2007) Thrombophilia and venous thromboembolism: implications for testing. Semin Thromb Hemost 33(6):573-581

6. Middeldorp S, van Hylckama VA (2008) Does thrombophilia testing help in the clinical management of patients? Br J Haematol 143(3):321-335
7. Bansal P, Factor V (2014) Leiden and MTHFR mutations as a combined risk factor for hypercoagulability in referred Patients population from Western India. Mol Cytogenet 7(1):P28

8. Perez-Pujol S, Aras O, Escolar G (2012) Factor V Leiden and inflammation. Thrombosis. 2012:594986

9. Simone B, De Stefano V, Leoncini E, Zacho J, Martinelli I, Emmerich J et al (2013) Risk of venous thromboembolism associated with single and combined effects of Factor V Leiden, prothrombin 20210A and methylenetethraydrofolate reductase C677T: a meta-analysis involving over 11,000 cases and 21,000 controls. Eur J Epidemiol 28(8):621-647

10. Ghumman GM, Ishtiaq R, Avasthi D (2021) Portal vein thrombosis in a patient with methylenetetrahydrofolate reductase gene mutation and normal homocysteine levels. Cureus. 13(9):e18225

11. Al-Maqbali JS, Al Rasbi S, Kashoub MS, Al Hinaai AM, Farhan $\mathrm{H}, \mathrm{Al}$ Rawahi B et al (2021) A 59-year-old woman with extensive deep vein thrombosis and pulmonary thromboembolism 7 Days Following a first dose of the Pfizer-BioNTech BNT162b2 mRNA COVID-19 vaccine. The American journal of case reports. 22:e932946

12. Thiele T, Ulm L, Holtfreter S, Schönborn L, Kuhn SO, Scheer C et al (2021) Frequency of positive anti-PF4/polyanion antibody tests after COVID-19 vaccination with ChAdOx1 nCoV-19 and BNT162b2. Blood 138(4):299-303

Publisher's Note Springer Nature remains neutral with regard to jurisdictional claims in published maps and institutional affiliations. 